\title{
7 \\ Generic Personal Communications Support for Open Service Environments
}

\author{
T. Eckardt ${ }^{*}$, T. Magedanz ${ }^{*}$, C. Ulbricht ${ }^{* *}$, R. Popescu-Zeletin ${ }^{* *}$ \\ ${ }^{*}$ Technical University of Berlin, ${ }^{* *}$ DeTeBerkom GmbH \\ ${ }^{* *}$ Voltastraße 5, 13355 Berlin, Germany, phone +49-30-4 67 01-0, \\ fax+49-30-467455, [ulbricht]@deteberkom.de
}

\begin{abstract}
The vision for future telecommunications is often described by the slogan ,information at any time, at any place, in any form “ driven by both the society's increasing demand for ,universal connectivity" and the technological progress in the area of mobile computing and personal communications. In order to realize this vision a Personal Communications Support System (PCSS) has been developed within a project of the R\&D-programme from the DeTeBerkom $\mathrm{GmbH}$, a subsidiary of the Deutsche Telekom AG, by Technical University of Berlin. The PCSS is designed to support advanced personal communications capabilities, i.e. personal mobility, service personalization, and advanced service interoperability, in a uniform way to numerous communication applications in distributed multimedia inhouse environments. From a functional perspective, the PCSS provides enhanced Intelligent Network (IN) and Universal Personal Telecommunications (UPT) capabilities with respect to customer addressing (based on logical names instead of numbers) and advanced customer control capabilities. From a design perspective, the centralistic IN/UPT approach to the realization of service logic has been replaced by a highly distributable, object-oriented approach based on X.500/X.700/Telecommunication Management Network (TMN) concepts. This paper presents the basic aspects of the PCSS, including design criteria, system functionality, platform design and evolution issues.
\end{abstract}

\section{Keywords}

Customer Control, Format Conversion, IN, Media Conversion, PCS, PCSS, Service Personalization, Service Interworking, TMN, UPT, User Mobility 


\section{INTRODUCTION}

The vision for future communications is ,information any time, any place, in any form", based on the idea of an open ,electronic" market of services, where an unlimited spectrum of communication and information services will be offered at different qualities and costs by different service providers. This service spectrum ranges from simple communication services up to complex distributed applications. In particular, new intelligent information services, such as hotel and flight reservation services are common place.

The prerequisite for this vision is global connectivity, based on a fast developing web of interconnected communication networks, comprising both fixed and wireless networks (Eckardt 1995). In addition, the provision of a global service infrastructure, based on networkindependent open service platforms is the other fundamental prerequisite, hiding the complexity of network diversity and allowing the fast and efficient creation, provision and management of future services.

This vision requires appropriate communication services and information systems, which are compatible with human communication processes. Users have to be supported in order to cope with the increasing complexity of global information availability and the increasing number of communication services which are based on different network technologies. Also the increasing reachability of people due to advanced mobile communication services requires adequate means for information filtering and communications control.

One important aspect of this vision is that the communication between people regardless of the communication service used should be based on person-oriented identifiers, such as the personal name or an individual personal number, rather than on service-specific terminal numbers, which becomes awkward in an environment of increasing communication service options. For example, there should be only one universal personal number for communicating with this user via phone, fax, paging, e-mail, video conferencing, etc. instead of having to use five quite different numbers and addresses.

Due to society's increasing demand for „universal connectivity“ and technological progress, mobile and personal communications are becoming fundamental attributes of future telecommunication systems. The trends toward personal communications can be viewed in terms of three areas:

\section{Mobility in fuxed and wireless networks}

Within the future communications environment users will have global access to an individual set of services, any time, any place and in any form. Hence the mobility of users will be enabled in the future environment by means of both terminal mobility and personal mobility. Terminal mobility and the corresponding wireless network interfaces and protocols (i.e. cordless, cellular and satellite) are fundamental for the provision of ,real“" global connectivity. It has to be stressed that besides voice the transfer of multimedia data becomes the ultimate target in wireless environments. This has severe impacts on the design of mobile multimedia terminals and the wireless networks capabilities. In addition, personal mobility will also enable global service access, allowing people to make use of any kind of terminal located at their whereabouts for obtaining access to their services. Since there exists a broad spectrum of terminals, e.g. POTS terminal versus multimedia workstation, services have to be designed to be accessed by terminals and networks with different capabilities. For example, users could generate by speech an e-mail from a public phone booth or from their PDA. 
Also session mobility will be supported in the future vision, allowing users to suspend a specific service session and later resume that very session from the same mobile or a different terminal. This means that the network will keep the context of a service session for a particular customer in order to allow moving people not only to have access to their own set of services but also to their last working session. In the context of personal and session mobility the notion of ubiquitous computing is important. In a target scenario it may be possible to allow mobile users for example within an organization, to use some ,blanc“ PDAs, which can be personalized after authentication in the user's working environment.

\section{Personalization of communication services access and delivery}

Personalization describes the customer's ability to define his own working environment and service working conditions stored in a „Personal Service Profile“. This profile defines all services to which the user has access, the way in which service features are used, and all other configurable communication aspects, in accordance with the user's needs and preferences, with respect to parameters, such as time, space, medium, cost, integrity, security, quality, accessibility and privacy.

\section{Interoperability of interfaces and services}

Interoperability is one step beyond personalization and describes the capacity of a communications system to support effective interworking between different services, supported by and offered on heterogeneous networks, with the long-term aim of achieving fully interworking applications.

The aspects of service personalization, service integration and interworking, enabling people to define if, when, where, for whom and in what form they will be reachable, are still in their infancy. Today no adequate standards exist for allowing people to define their own individual communications and services environment, referred to as ,personal services communications space" (Guntermann 1993). However, advanced customer control capabilities are required for the realization of real personalized communications.

In this context standards in the field of Intelligent Networks (IN) (ITU-T Q.1200) and Universal Personal Telecommunications (UPT) (ITU-T F.851) provide only limited capabilities without reflecting the emergence of Telecommunications Management Network (TMN) standards (ITU-T M.3010) in the context of service management. This problem has led to many research activities in the field of IN/TMN integration (Magedanz 1995, 1995a), where one thesis promoting the use of TMN concepts for providing IN service capabilities is gaining increasing attention.

Based on these considerations, in 1993 the DeTeBerkom GmbH, a subsidiary of the Deutsche Telekom AG, has initiated a project within its R\&D-programme in the field of IN/TMN integration with the focus on personal communications support and evolution towards future object-oriented service platforms, such as the Telecommunications Information Networking Architecture (TINA) (TINA 1995). In the course of this project, a TMN-based Personal Communications Support System (PCSS) (Eckardt 1994, Eckardt 1995a, Magedanz 1995) has been developed, which provides advanced personal communications capabilities within distributed multimedia inhouse environments. 


\section{OVERVIEW OF THE PERSONAL COMMUNICATIONS SUPPORT SYSTEM}

The Personal Communications Support System (PCSS) has been developed to provide generic capabilities for personal mobility, service personalization, and advanced service interoperability in a uniform way to numerous communication applications, ranging from simple telephony up to multimedia conferencing services. From a functional perspective, the PCSS provides enhanced UPT capabilities with respect to customer addressing (based on logical names instead of numbers) and advanced customer control capabilities. From a design perspective, the centralistic Intelligent Network approach to the realization of service logic has been replaced by a highly distributable, object-oriented approach.

\subsection{The PCSS Approach}

The PCSS approach differs from the conventional Intelligent Network (ITU-T Q.1200, ITU-T F.851] approach in a number of elementary points:

The PCSS generally uses personal references as communication addresses instead of physical addresses such as terminal IDs or network access points. The PCSS user is relieved from the need to configure a personalized call management in terms of originating and destination terminal addresses presuming an exact and fixed user-terminal relationship. In fact, in scenarios of nomadic personal communications, the fixed user-terminal relationship is replaced by a a flexible, dynamically changing association between nomadic users and various terminals in the current proximity of the user. The general goal of the PCSS approach is to bring a uniform, user-centric, i.e. non-technical approach to communications. It replaces terminal-oriented by person-oriented communications.

The PCSS provides capabilities for a uniform, service-neutral configuration and personalization of all types of teleservices subscribed to or used by a user. The advantage of this approach is a more focused, integrated view of the control, configuration and management of the total communications environment offered to the customer.

Similar to UPT, the users have to register at each new location in order to indicate to the network where to route a call directed to their personal number. But the PCSS user registers at locations, not at terminals. In this regard, we defined several registration procedures to be supported by the PCSS: automatic registration - that is an automatic user tracking applying Active Badges (Want 1992, Harter 1994) developed at Olivetti Research Ltd. in Cambridge UK, manual registration, and scheduled registration using a kind of electronic personal diary stored in the network.

\subsection{PCSS Functional Model}

The main dynamic mapping function within the PCSS is a multi-stage mapping of a PCSS address or personal identifier of the called party to an appropriate physical device address of the Customer Premises Equipment (CPE) at the user's temporary location. It is characterized by a distinctive model for the dynamic CPE selection defining a selection process in 4 stages (see Figure 1):

- The evaluation of a user's call logic constitutes the $I^{\text {st }}$ stage of the multi-stage dynamic CPE selection process. It provides the management of the user's reachability. In case of 
call forwarding it represents a person to person mapping, otherwise one of the following features will be chosen: call accepting, call blocking, announcement, voice box, etc..

- At the $2^{\text {nd }}$ stage a mapping of the user's identifier to the location is made based on user registration data if the exact recipient of the communications invitation has been settled and no further call management will be performed.

- The $3^{\text {rd }}$ stage performs a mapping from a location to a virtual communication endpoint corresponding to a group of terminals, i.e. Virtual Access Point (VAP). A VAP encompasses knowledge on terminal capabilities, supported services, etc. and a selection mechanism.

- The $4^{\text {th }}$ stage selects an appropriate terminal ID from the group of devices contained by the VAP. This function will be performed by the Virtual Access Point selected in the previous stage. The processing at this stage is parameterized by a service type, used media, and optionally by user preferences.

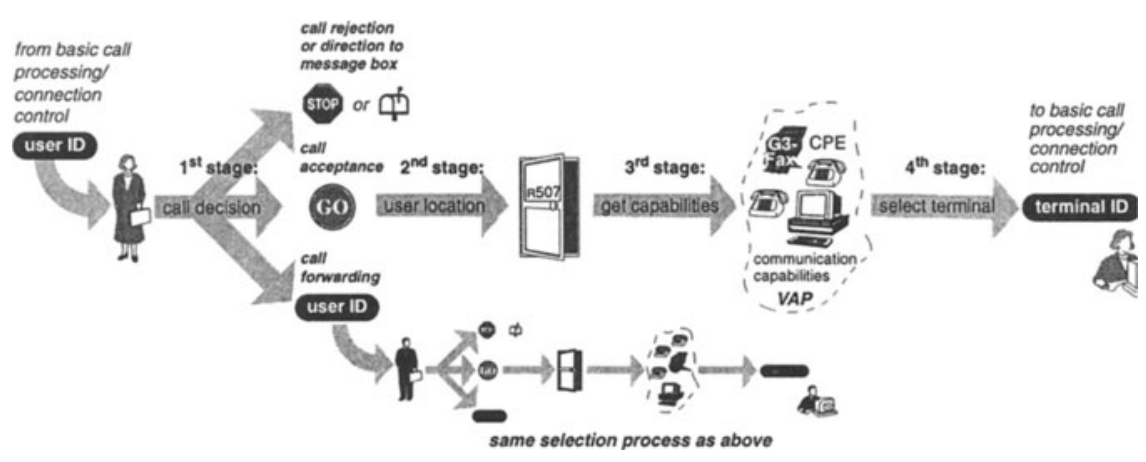

Figure 1 PCSS mapping of user ID to terminal ID

\subsection{PCSS Object Model}

The PCSS component model has been defined to structure the functionality required for the multi-stage PCSS call processing into distinctive functional components. This component model defines a number of functional components and elementary objects that are required to provide the PCSS call processing. The term component is used to denote a distributable server, a management subsystem or a X.500 DSA which provide the functionality of a „host“ for PCSS objects and profiles. The units of individual state, interaction and addressing are termed objects, e.g. the Call Session, Call Logic Agent, Registration Agent, Virtual Access Point, Service Access Point. Two of these objects are called ,agent "“ since they represent and act on behalf of an individual user. The components may be understood as a kind of container or process for multiple objects of a specific type. The hosting components for "agents“ are called „server" and all other hosting components are called „manager" or „MIB“.

1. Please note, that the term 'agent' used in this context is not to be dismissed as an agent in the X.700 CMIP context. 
Figure 2 gives an overview of the basic components of the PCSS which cooperate in order to provide the PCSS call processing. We applied an object-based modelling that defines the individual elementary functional components of the PCSS component model in terms of the operational interfaces they provide.

The interface descriptions of the functional components Call Logic Server and Registration Server are specified according to the Guidelines for the Definition of Managed Objects (ITU-T X.722). These functional components are modelled and implemented as management systems. Each of the components contains a number of dedicated Managed Objects (MOs) in specific PCSS Management Information Bases (MIB). Besides these X.700 components, a number of profile stores are indicated, which have been realized as X.500 DSAs, e.g. user profile store, zone profile store, virtual access point store, and service access point store. These different profile stores may be arbitrarily distributed over one or multiple DSAs.

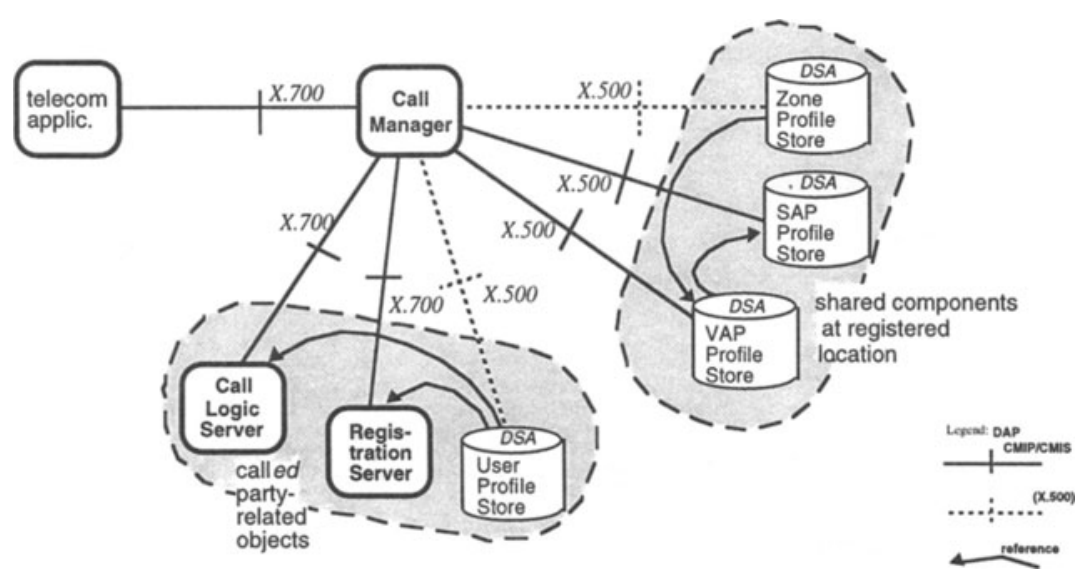

Figure 2 Basic components of the PCSS

The Call Manager contains and maintains a set of Call Sessions. A Call Session is an object representing the state of a particular PCSS call. The Call Logic Server incorporates multiple Call Logic Agents with each of the agents representing the personalized call logic of a single user. The third component, the Registration Server contains multiple Registration Agents that represent the different registered users within that location area which is handled by the Registration Server. Currently, the Virtual Access Points (VAPs) profiles and Service Access Points (SAPs) profiles are passive objects, i.e. X.500 directory entries. Two of the active components, the Call Logic Server and the Registration Server, have been realized as dedicated management processes comprising a CMIS agent and multiple MOs representing the Call Logic Agents and Registration Agents.

Figure 3 outlines the basic interactions of the objects above described, required to provide the PCSS Call Processing. The activity of calling a party (0) within a teleservice results in the request for the creation of a Call Session object dedicated to that particular call (1). It coordinates and provides the total multi-stage PCSS Call Processing. The called party addresses the Call Session Object with the personal reference of the called party in order to receive a physi- 
cal address where the called party can be reached. At the lower left, the Call Logic Agent of the called party is shown. The Call Session object requests the evaluation of the Call Logic Agent (2). In this scenario the evaluation results in a "Call Forwarding" to a different person. This may happen numbers of times: multiple Call Logic Agents are addressed during a chained, multiple Call Forwarding (2a-n). Finally, the Call Logic Agent of the last addressed party is assumed to accept the call. In the next step the corresponding Registration Agent will be approached by the Call Session object to retrieve that last party's current location (3). The Registration Agent contains the information on the user's current location in terms of a Zone ID. This is a link to the Zone Profile that holds any required information on the location, especially the reference to the correspondingVirtual Access Point (VAP) (4). Each VAP represents a number of Service Access Points (SAPs) that contain information on specific communication endpoints and terminals at a certain location. The VAP will dynamically select an appropriate SAP (5a) thereby considering the state of the terminal (i.e. busy or idle) (5n). The final SAP including the proper communication address will be replied (4) to the Call Session object and finally to the Basic Call Process in the requesting teleservice $(4,6)$. Subsequently, the Basic Call Process can establish a connection to the resulting Terminal ID given by the $\mathrm{SAP}_{x}$.

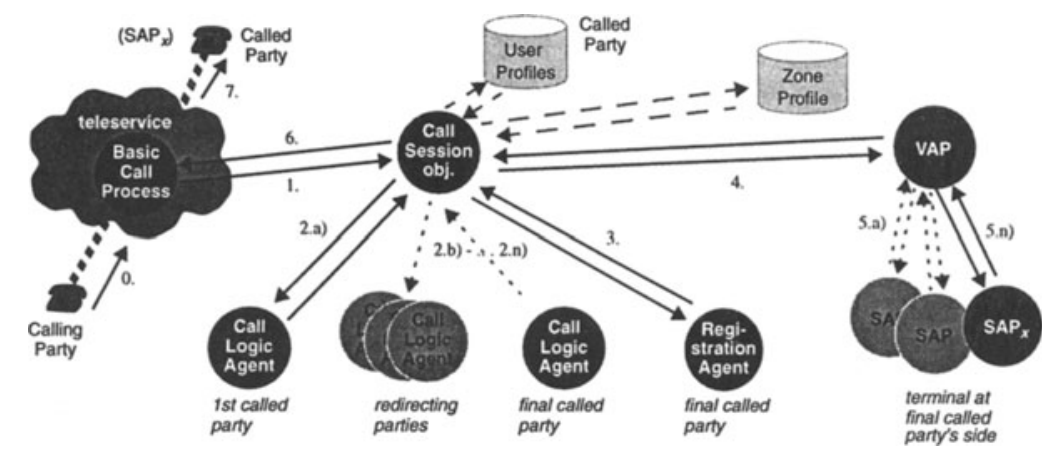

Figure 3 Object interactions within the PCSS Call Processing

\subsection{PCSS Platform}

When designing the PCSS, we concentrated on a uniform modelling of PCSS functionality completely based on current X.500 Directory, X.700 Management, and Telecommunications Management Networks (TMN) standards. The underlying idea has been: to use concepts of OSI/TMN management for both service management and service control to enhance basic telecommunications services to the level of IN services or Service Features.

The uniform application of object orientation and management concepts throughout the total design allowed us to develop a systematic and regular overall model or ,umbrella“ for personal communications resulting in a PCSS Platform supporting a wide range of applications. This PCSS Platform (shown in the centre of Figure 4) comprises a PCSS Applications Framework built on top of an advanced management platform ( RACE H.430, 1993) integrating X.500 and X.700. The management system accesses specifically defined PCSS User Profiles via X.500 Directory User Agents (DUAs) applying the Directory Access Protocol (DAP). 
Additionally, X.700 CMIS/CMIP managers are used for a location transparent access to dynamic PCSS Managed Objects (e.g. Registration, SAPs, VAPs) using a naming space established by the X.500 Directory Service (Tschichholz, 1993).

The PCSS Applications Framework on top of that management platform is the basic interface to the PCSS data. It consists of an extensive set of objects which provide access to all information and data stored within the scope of the PCSS profiles and services. The main purpose of this completely object-oriented interface is to serve as a common foundation for applications of any complexity. The application framework and its various component objects support various telecommunications services. At the same time, it enables a rapid development of PCSS-specific management applications, e.g. for user registration or user profile management, and various kinds of personal communications-related multimedia applications.

Every PCSS application is based on the retrieval and modification of data stored in the profile databases or managed by management services within the scope of the PCSS. The main purpose of the PCSS application framework is to provide a uniform and intuitive but nevertheless powerful interface to PCSS related information for all existing and forthcoming front-end and helper applications. The most important of these front-end applications are the User Profile Management Service (UPMS) and the User Information Service (IVIS), which are described below.

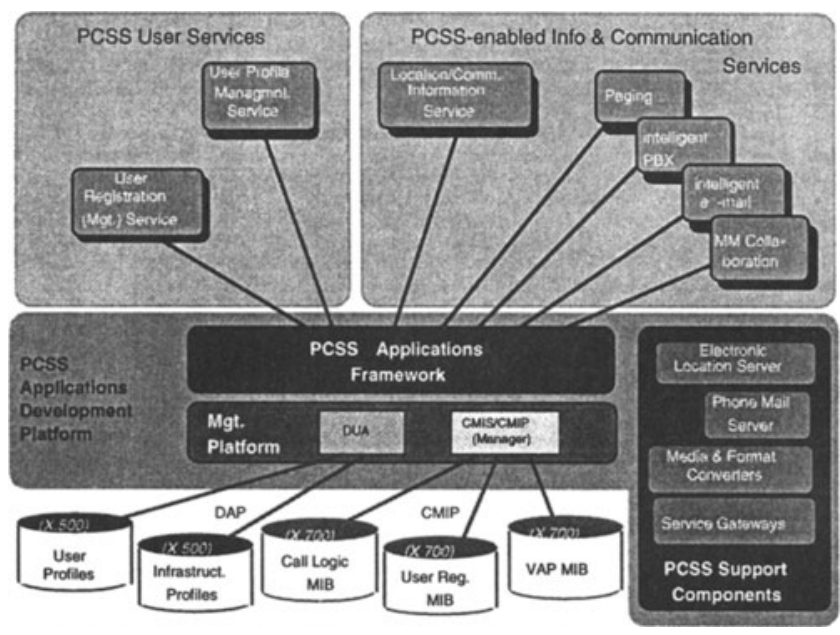

Figure 4 PCSS prototype platform

\section{USER PROFILE MANAGEMENT SERVICE}

While the PCSS application framework supports the low level data access, it does not provide a graphical user interface to personal data tailoring and management. Therefore, a graphical user interface based front-end application is needed for the PCSS users to view and edit the 
contents of their own, personal user profiles. It must enable the users to easily adapt the user specific information stored within their personal Generic Service User Profile (GSUP). By this way, this PCSS front-end application empowers the users to customize the rich set of user related services provided by the PCSS and to suit the PCSS communication environment and the provided PCSS service functionalities to individual preferences.

Due to the highly complex PCSS information model and the various service features provided, it is essential for this application to support the user with an intuitive and easy to use graphical user interface. The front-end application has to abstract the several complex information structures and to simplify their visualization, and it has to support the users in their interaction with the application. It must protect the users from making mistakes, and it must offer support if the users nevertheless encounter errors.

The User Profile Management Service (UPMS) offers all the capabilities required to enable the PCSS users to adapt their personal data and to customize their individual PCSS communication environment. To fulfil these needs, the UPMS provides the following functionalities for the manipulation of the contents of the personal GSUP to the PCSS users:

- user authentication to ensure secure access to user profiles,

- user profile configuration for the modification of personal user data,

- user registration for the manual registration of current location information,

- personal schedule configuration for automated registration of location data by schedule, and

- communication configuration for customization of the personal call logic.

These several features of the UPMS front-end application are realized by a set of basic application components, which can be considered as application building blocks for the realization of the UPMS. They are described below in more detail.

\subsection{User Identification and Authentication check}

The connection and access to the PCSS database must be strictly secured to protect the confidential personal information contained within the Generic Service User Profile (GSUP) and the other related infrastructure and organizational profiles as well as to the registration information against access by unauthorized users. Therefore, the PCSS users have to pass a PCSS identification and authentication procedure successfully before using the UPMS.

The UPMS users do not only have to give their unique identification for access to the PCSS, but they also have to enter an individual password matching the given identifier. The users are granted access to the PCSS and the UPMS only if they enter the correct password. Unlike the personal identifier, this individual password can be defined by the users, of course. It is stored in encrypted form as part of the general user data within the scope of the personal GSUP.

\subsection{Personal User Data Editor}

The personal Generic Service User Profile of all PCSS users contains basic, service independent information. This information, especially the general user data, are not directly related to the several communication services provided by the PCSS, but nevertheless they are part of the individual PCSS user environment. Therefore, the personal user data editor enables the users to customize these data. 
The general user data like common name, surname, and private address, the personal title and organizational status can easily be viewed and modified by the users (see figure 5).

The PCSS system supports the modelling of complex organizational structures within companies. It enables the management of service access points, zones, events, and individuals, and also the definition of groups containing references to these objects. So departments or projects as well as any other assembly of PCSS objects can easily be represented.

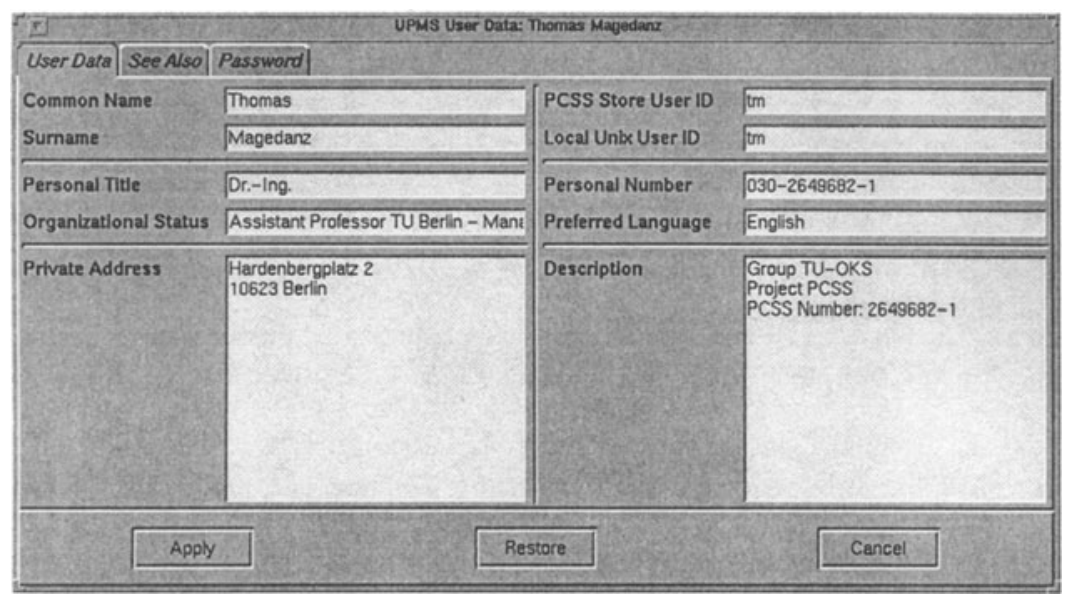

Figure 5 Personal User Data Editor Module of the UPMS

The UPMS users can use the personal user data editor to manage other available objects or groups of objects within the scope of their personal GSUP, hidden below the menu button see also. The users can tailor their personal list of references by selection from lists of available individuals, groups and events. The editor provides simple functionalities to rearrange the list as well as to add references to or remove them from the list. Within the scope of the personal user data editor, the users are also able to replace their password needed for secure authentication to the PCSS.

\subsection{User Registration Manager}

According to the user oriented approach to communication by the PCSS, abstract user identifiers must be mapped to physical communication addresses. Therefore, the PCSS has to gather needed information about the current location of the participating users. The location data is managed by the PCSS registration server of the PCSS X.700 management services. The personal GSUP contains references to these location information.

The PCSS registration server can retrieve current location information from different sources. Unlike the location information to be gained by automatic registration, the user can individually define location information via the user registration manager.

The manual registration procedure enables the user to provide the PCSS with his current location data manually. The registration manager provides the users with a list of all available 
zones from which they can select their current location. The user registration manager selects a location for the users. This default choice for the manual registration can be, in this precedence, the location the users are currently registered to, the users specified default location or the location of the terminal actually used for registration via the UPMS. In addition, the users are offered a list of recently registered locations for easy retrieval of location information. For convenience, the manual registration procedure allows the users not only to specify their real current location for registration, but also each other location within the scope of the PCSS.

\subsection{Personal Schedule Editor}

The registration server manages current location information registered manually by the PCSS users via the user registration manager. Unlike the current location information depending on the presence of the users and the use of the UPMS, the personal schedule enables the users to specify their future locations during absolute or even periodically recurring time intervals independently of the registration server.

The personal schedule is described by a scheme of rules. Each of these personal schedule rules describes a time interval and an associated future location. During their evaluation by the PCSS, the schedule entries are assigned priorities according to their order in the personal schedule. When looking for the location associated with a certain time interval, it is matched against the time frames contained in each schedule one after another. The first schedule entry matching the given time interval then determines the current location.

The PCSS users must be enabled to setup and to configure the contents of the personal schedule to meet their individual requirements. Therefore, the UPMS supports the users in the customization of their personal schedule. Using the graphical user interface provided by the personal schedule editor, the users can define and modify the entries of the personal schedule. Assigning locations to time intervals, the users can describe events or appointments taking place once or regularly as well as future locations assigned to certain complex time intervals.

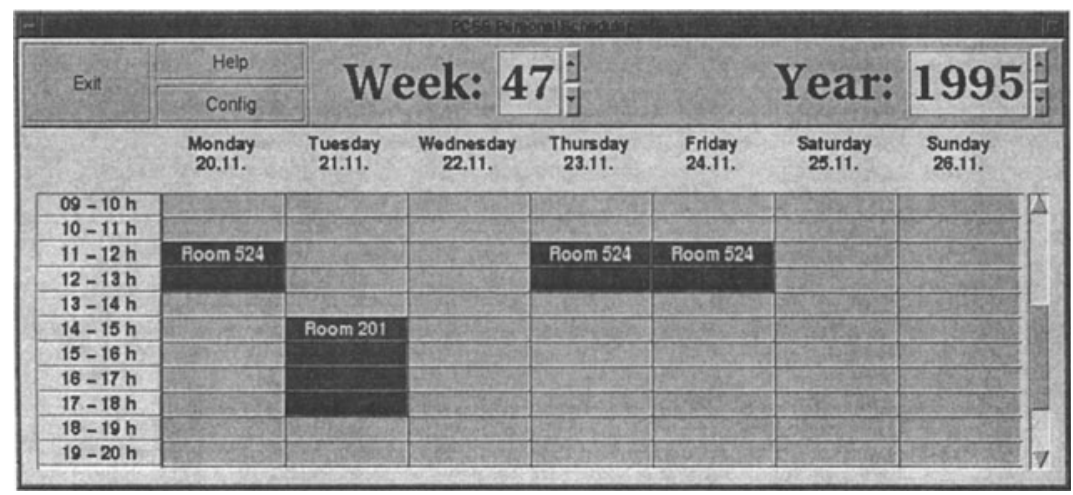

Figure 6 Weekly Schedule Plan

By dragging the mouse within the actual week plan, the users can highlight a number of adjacent schedule fields to create a new schedule entry. Following a dialogue allows to create a 
new schedule entry by specification of a time interval and the assignment of a location.

For periodically recurring time frames, an optional type of repetition as well as the end of its iteration can be given. There are several different types of repetitions selectable by the user. By using these repetitions, frequently visited locations can be indicated. No Repetition is the default, but repetitions for every week, every month, every year, week in month or day in week are possible to choose.

\subsection{Call Logic Editor}

The most important capabilities provided by the PCSS are the mapping from abstract personal identifiers to physical communication addresses and the advanced incoming call management features. These high level PCSS functionalities are performed by the PCSS call manager component. Concerning the address resolution and the incoming call processing performed by the PCSS, the most important information are the data stored within the personal call logic.

Similar to the personal schedule, the personal call logic is described by a scheme of user defined rules. These rules determine the personal call logic matrix. The rules contain instructions for the handling and processing of calls incoming to the PCSS. Each call logic rule contains actions to be executed if an associated set of conditions is matched by the actual parameters of incoming calls. Using the various different supported condition types, the evaluation of incoming call handling actions can depend on the time of the incoming call, on the calling user or device, and on the requested communication medium. Furthermore, all conditions can be combined to even more complex compound conditions.

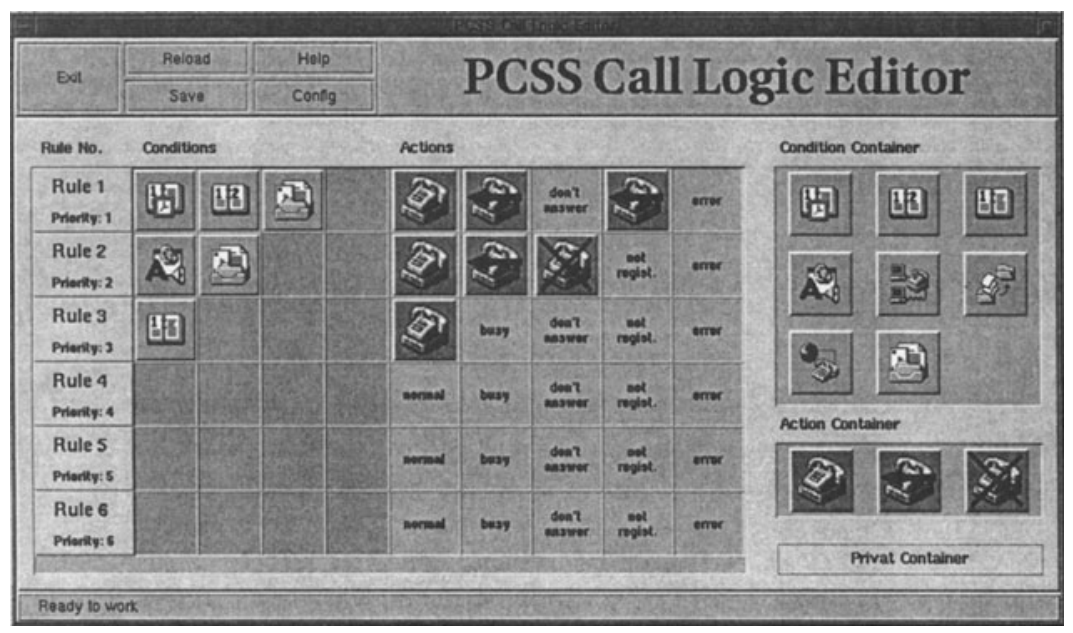

\section{Figure 7 Call Logic Editor}

During the processing of incoming calls by the PCSS, the personal call logic of the recipient is evaluated by the PCSS call manager. The priorities are assigned to call logic rules according to their position in the call logic matrix. When searching for the actions to be performed on the incoming call, the conditions specified in each rule are matched against the actual call 
parameters. The first rule matching all given conditions determines the set of actions to be executed.

For the setup of an individual communication environment within the scope of the PCSS, the UPMS must enable all PCSS users to customize the service specific information contained in their personal GSUP. Because its significant effect on the call processing by the PCSS, one of the main aims of the UPMS is to support the users in the setup and configuration of their personal call logic. The call logic editor provides the PCSS user with a powerful and easy to use graphical interface for the setup and customization of the personal call logic matrix.

\section{USER INFORMATION SERVICE}

The idea behind the realisation of an „User Information Service“ application is to use the services and information managed inside the PCSS not only for the purposes of the PCSS and its participants themselves, but also to make the core functionality and data visible to any participating user or external users outside the PCSS. A first approach to this idea is the realisation of an Interactive Visitor Information Service (IVIS), which responds to the needs of a visitor of getting information and contacting a company or organisation and their individuals in a fast and efficient way.

In the given scenario of an organisation which uses the PCSS environment for its inhouse communication, the implementation of IVIS will realise a kind of „electronic receptionist“, which provides a visitor with a set of "functionalities" as in real life, combining it with the information managed inside the PCSS. The task of connecting a visitor and a person which corresponds to the needs of the visitor is performed interactively with the visitor using the application IVIS. Therefore one major task of the application IVIS is the search for a person represented in the databases of the PCSS. During the process of searching the user will be supported by a highly intuitive and simple user interface through the application IVIS.

The first prototype of the application IVIS will only act as a ,passive " member of the PCSS architecture and interacts with the user like a common information system. Therefore IVIS uses the contents of the different PCSS Profiles, e.g. the User Profiles, as a smart database and presents this information to the user in a easy understandable way.

The application IVIS will lead a visitor through the „universe“ of the organisation by representing their people, their locations, current events, etc. Any visitor who wants to meet one specific individual inside the company will be supported by the application IVIS and the PCSS in presenting information about a person, who matches exactly the visitors needs and requirements. Using the up-to-date information stored inside the PCSS database, a visitor will be led by the application IVIS in a simple and intuitive way without involving a third person in this process. Her interactions will be supported with the presentation functionalities of IVIS described above by showing photos, displaying textual descriptions, etc. Additionally, the visitor will be provided with actual location information of the desired people and with information how to reach this location through a map or floorplan. By viewing the information about the employees of an organisation, the visitor will also be provided with a couple of alternate persons and related topics about any specified individual. This will prevent him from additional complex and time consuming searches inside the PCSS, if the desired partner is not available or does not want be disturbed by a visitor. 
In the following example there was be selected the button „PCSS“ inside the search screen containing the ,projects“. The list of buttons now represents all individuals related to the project PCSS, including a small photo of each person. Additional textual information about the currently selected project is presented to the user in the upper part of the screen.

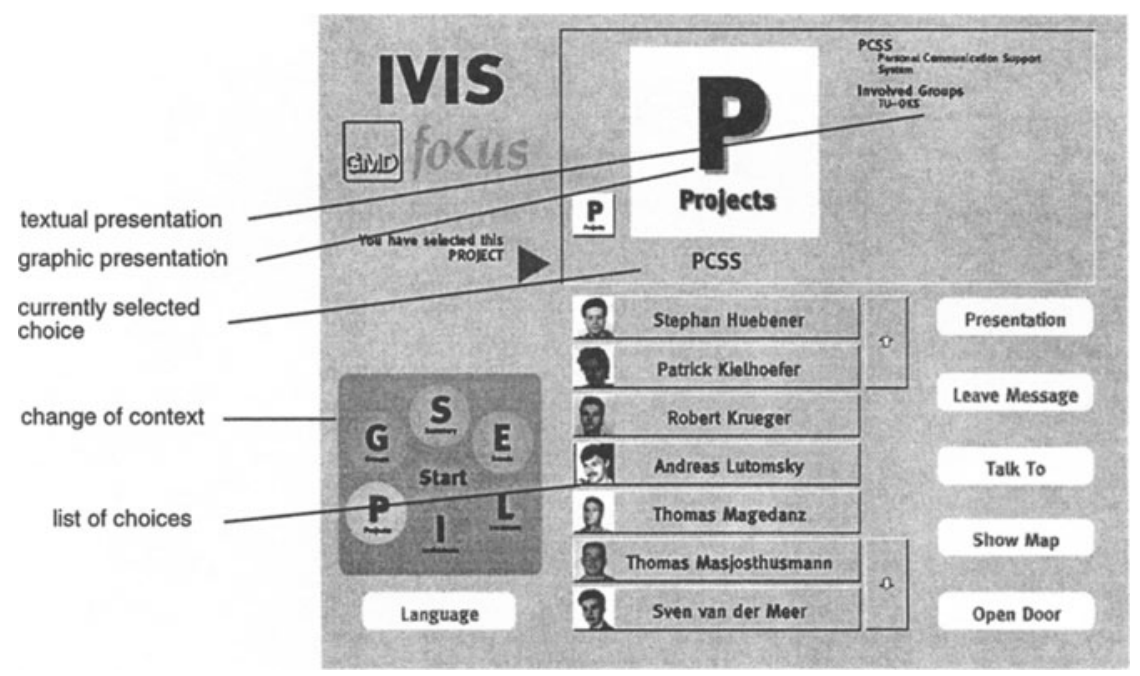

Figure 8 Contents of the Search Screen of IVIS

Beneath the area of the search additional interaction-components will allow the user to invoke other and future components of the PCSS like ,intelligent map“, communication, or more generic functionality like printing, language-selecting, etc. Another group of interaction elements besides the list of selectable choices of the search will show a small representation of the start screen with the same functionality. The characteristic look of this group makes it easy for a user to associate its purpose. So a visitor may freely change between the different main contexts without returning to the start screen. If the user changes his current context inside the search, e. g. from groups to project the application IVIS will save the contents of the current context for a later use. So a user does not need to start the search new from the beginning, if he is temporarily changing the context.

\section{PCSS EVOLUTION}

Current enhancements of the outlined PCSS concentrate on dynamic media type and format conversion required for entirely supporting the above outlined vision of ,information at any time, at any place, in any form“. Therefore, a powerful set of information converters, including both format and media converters, which can be dynamically configured according to a given personal communications scenario are under development (Magedanz 1996). 
Furthermore, another point of PCSS evolution is the migration from a TMN architecture towards TINA architecture [TINA-95]. In this context the PCSS has become recently an auxiliary project of the TINA consortium work, labelled „PCS in TINA“. The basic objective of this TINA auxiliary project is the incorporation of the developed PCSS concepts into the TINA service architecture in order to support advanced personal communications within TINA (Eckardt 1996).

\section{ACKNOWLEDGEMENTS}

The following persons have contributed to the development of the presented PCSS: S. Arbanowski, F. Gadegast, L. Hagen, S. Huebener, S. van de Meer, U. Scholz, M. Vetter, H. Wang.

\section{ACRONYMS}

CMIP Common Management Information Protocol

CPE Customer Premises Equipment

DAP Directory Access Protocol

GSUP Generic Service User Profile

ID Identifier

IN Intelligent Network

IVIS Interactive Visitor Information Service

MIB Management Information Base

MO Managed Object

PCS Personal Communications Support

PDA Personal Digital Assistent

PCSS Personal Communications Support System

SAP Service Access Point

TINA Telecommunication Information Networking Architecture

TMN Telecommunications Management Network

UPMS User Profile Management Service

UPT Universal Personal Telecommunications

VAP Virtual Access Point

X.500 Directory

X.700 TMN / OSI Management 


\section{REFERENCES}

Eckardt, T. and Magedanz, T. (1994) On the Personal Communication Impacts on Multimedia Teleservices. International Workshop on Advanced Teleservices and High-Speed Communication Architectures (IWACA), Heidelberg, Germany, September 26-28, 1994.

Eckardt, T. and Magedanz, T. (1995) On the Convergence of Distributed Computing and Telecommunications in the Field of Personal Communications. Kommunikation in Verteilten Systemen (KiVS), Zwickau, Germany, February 22-24, 1995.

Eckardt, T. and Magedanz, T. (1995a) The Role of Personal Communications in Distributed Computing Environments. 2nd Interantional Symposium on Autonomous Decentralized Systems (ISADS), Phoenix, Arizona, USA, April 25-26, 1995.

Eckardt, T. and Magedanz, T. and Stapf, M. (1996) Personal Communications Support within the TINA Service Architecture - A new TINA-C Auxiliary Project. Proc. of 6th TINA Conference, Heidelberg, Germany, September, 1996.

Guntermann, M. et.al. (1994) Integration of Advanced Communication Services in the Personal Services Communication Space - A Realisation Study. Proceedings of the RACE International Conference on Intelligence in Broadband Service and Networks (IS\&N), Paris, France, November 1994.

Harter, A. and Hopper, A. (1994) A Distributed Location System for the Active Office. IEEE Network, Special Issue on Distributed Applications for Telecommunications, January 1994.

ITU-T Draft Recommendation F.851 (1991) Universal Personal Telecommunications - Service Principles and Operational Provision. November 1991.

ITU-T Recommendation M.3010 (1992) Principles of a Telecommunications Management Network. Geneva, 1992.

ITU-T Recommendation X.722 (1992) Information Processing - Open Systems Interconnection - Structure of Management Information - Part 2: Guidelines for the Definition of Manged Objects. Geneva, 1992.

ITU-T Recommendations Q.1200 series (1992) Intelligent Network. Geneva, March 1992.

Magedanz, T. and Popescu-Zeletin, R. and Eckardt, R. (1995) A (R)evolutionary Approach for Modeling Service Control in Future Telecommunications - Using TMN for the Realization of IN Capabilities. International TINA Conference, Melbourne, Australia, February $13-16$, 1995.

Magedanz, T. (1995) On the Integration IN and TMN - Modeling IN-based Service Control Capabilites as Part of TMN-based Service Management. 6th IFIP/IEEE International Symposium on Integrated Network Management (ISINM), Santa Barbara, California, USA, May 1-5, 1995.

Magedanz, T. and Pfeifer, T. (1996) An Intelligent Personal Communications Support System enabling Information any time, any place, in any form. Submitted to IEEE Global Telecommunications Conference, London, United Kingdom, November 18-22, 1996.

RACE Common Functional Specifications (CFS) H.430 (1993) The Inter-Domain Management Information Service (IDMIS), Issue D. December 1993.

TINA-C Doc. No. TB_MDC.018_1.0_94 (1995) Overall Concepts and Principles of TINA. February 1995.

Tschichholz, M. and Donnely, W. (1993) The PREPARE Inter-Domain Management Informa- 
tion Service. RACE IS\&N Conference, Paris, France, 1993.

Want, R. and Hopper, V. and Falcao, V. and Gibbons, J. (1992) The Active Badge Location System. ACM Transactions on Information Systems, Vol. 10, No. 1, 1992.

\section{BIOGRAPHY}

Cordula Ulbricht finished her studies in computer sciences at the Technical University of Berlin in 1994. Since then she has been working as a project manager at the DeTeBerkom GmbH, a subsidiary of the Deutsche Telekom AG, in the field of Open Communications Systems, Intelligent Networks and TINA. Presently her work focuses mainly on the development of future telecommunication services considering the aspects of mobility. 